\title{
REACCIONES EN LA PRECIPITACIÓN ANTE OSCILACIONES OCEANICAS EN SUS TERMPERATURAS SUPERFICIALES: DEPRESIÓN TECTÓNICA CENTRAL, COSTA RICA
}

Marvin E. Quesadal marvin.quesada@ucr.ac.cr

Matt Marsik2mmarsik@ufl.edu

\section{RESUMEN}

Se analiza la influencia del evento el ENOS en la precipitación de la Depresión Tectónica Central de Costa Rica, encontrándose que dicha respuesta es diferente, dada la diferencia de temperaturas superficiales entre océano Pacífico y Atlántico. Estos pueden contribuir en mayor o menor grado en la producción de masas de aire cargadas de humedad que puedan llegar hasta esta región. Similarmente, dicha región presenta irregularidades geomorfológicas en las dos principales subcuencas Virilla y Grande de San Ramón que contribuyen aún más con las diferencias en los niveles de precipitación, siendo esta reducción en la precipitación aún mayor en la subcuenca del río Grande de San Ramón.

Palabras clave: ENOS, subcuencas, Virilla, Grande de San Ramón, precipitación, vientos, caudales, Costa Rica.

PRECIPITATION REACTIONS DUE TO OCEANIC OSCILLATIONS IN ITS SURFACE TEMPERATURes: CENTRAL TECTONIC DEPRESSion, Costa Rica

\section{ABSTRACT}

It is analyze the response of the ENSO event regarding the precipitation in the Central Tectonic Depression of Costa Rica. It was found that such response is different given the variation in sea surface temperatures among the Pacific and the Atlantic Ocean. These may contribute, in varying degrees, to the formation of air masses loaded with humidity which may reach this region. Similarly, such a region presents geomorphologic irregularities in two main sub-basins that contribute even more regarding the differences in the levels of precipitation; this reduction is greater in the subasin of the Grande de San Ramón River.

Key words: ENSO, Basins, Virilla, Grande San Ramon, precipitation, winds, flows, Costa Rica. 


\section{INTRODUCCIÓN}

Costa Rica muestra una gran riqueza hídrica dado que existe una gran diversidad en sus características climáticas y orográficas, lo que ha permitido que el agua se almacene en zonas montañosas y luego escurra por los ríos hasta las zonas costeras, permitiéndose un aprovechamiento en distintas actividades humanas. Sin embargo, en los últimos años la Depresión Tectónica Central ha sufrido un acelerado proceso de expansión urbana. Dicho crecimiento urbano supone grandes presiones sobre ciertos servicios básicos y un fuerte impacto sobre el entorno natural. Dentro de estos impactos, uno de los más serios es el que se ha ejercido sobre el régimen hidrológico. Unido a ello, en ciertos años los caudales de los ríos se ven disminuidos o aumentados a causa de variaciones de índole climatológica.

Varias investigaciones científicas han analizado la interrelación entre la variabilidad hidroclimática y el evento denominado el ENOS (El Niño y/o La Niña-Oscilación del Sur) (CAVAZOS, T., GOCHIS, D., MARENGO, J., \& BOULANGER, J.-P, 2008; MURPHY J., K. GEORGAKAKOS \& E. SHAMIR, 2013). Desde una amplia perspectiva, los caudales y la precipitación podrían tener consecuencias directas en una variedad de aspectos económicos, sociales, ambientales cuando son sometidos a variaciones climáticas. Algunos investigadores (Hastenrath, 1990: Poveda y MeZA, 1997; George eT AL., 1998; MAGAÑA ET AL., 1999; ENFIELD AND ALFARO, 1999; W AYLEN AND QUESADA, 2002; DURAN A., J. GEMENO, J.A. AMADOR \& R. NIETO, 2010), han hecho estudios sobre el comportamiento del ENOS, en países tropicales de América. Algunos de ellos han analizado su relación a través de registros de caudales y precipitación.

El ENOS manifiesta en algunas regiones excesos de precipitación y en otras mermas (YOULTON ET AL, 2012; POVEDA ET AL, 2013; ENRIQUEZ ET AL, 2014). En Costa Rica en la vertiente Pacífica, durante su fase fría denominada La Niña se presentan abundantes precipitaciones, produciendo incluso inundaciones especialmente en regiones urbanas y agrícolas. En tanto, durante su fase cálida denominada El Niño son mermas en la precipitación, lo que ha afectado los usos de las aguas, especialmente para uso hidroeléctrico. Por ejemplo, una de las compañías eléctricas de Costa Rica (ICE) obtiene un $70 \%$ de su energía eléctrica a partir del agua, incluso la mitad de ella es generada en una represa que se denomina Arenal (GEORGE ET AL., 1998). Asimismo, son varios los países latinos que han tenido que recurrir a los llamados "apagones" como medida para ahorrar energía, 
especialmente durante la época seca, tal es el caso de Colombia, Perú, Nicaragua y Costa Rica, entre otros., inclusive durante los cinco primeros meses del año 2010, dicha eventualidad ocurrió en Venezuela, uno de los países con mayores reservas de petróleo del continente Americano, pudiendo destinar combustibles fósiles para mover las turbinas en lugar de agua, prefirieron ahorrar energía por medio de las suspensiones periódicas del fluido eléctrico.

Esta investigación muestra un interés científico y práctico dado que eventualmente puede constituir el punto de partida en la planificación integral de una región ante posibles extremos de precipitación. Esto a su vez podría causar crecidas e inundaciones intensas en ciertos años y en algunos periodos del año. Además, es un ejemplo para la elaboración de predicciones climatológicas y previsiones en las distintas actividades humanas como la agricultura, la cual se puede ver afectada ante variaciones de intensidad y la dinámica del ENOS y otros eventos meteorológicos parecidos.

\section{UBICACIÓN DE LA CUENCA EN ESTUDIO}

La parte alta y media de la cuenca del río Grande de Tárcoles (Subcuencas Virilla y Grande de San Ramón) se ubica en la Depresión Tectónica Central de Costa Rica, donde se localizan tres de las ciudades más pobladas del país y varias ciudades secundarias de importancia, no sólo por su crecimiento demográfico, sino también por sus actividades económicas. Esta cuenca limita al Norte y al Este con la Sección Cordillera Volcánica Central, la cual alcanza altitudes de hasta 3440 m.s.n.m.

Por el Sur se encuentran los cerros de Escazú que tienen altitudes cercanas a los 2000 m.s.n.m. En el Oeste, la cuenca presenta una abertura producto del cañón del mismo río, el cual luego desemboca al océano Pacífico. La cuenca alta y media se divide básicamente en dos sectores; el oriental que es drenado por el río Virilla y el occidental por el río Grande de San Ramón, estos a su vez cuentan con una serie de tributarios que al unirse forman la cuenca del río Tárcoles (Figura 1). 
Figura 1. Ubicación del Área en Estudio

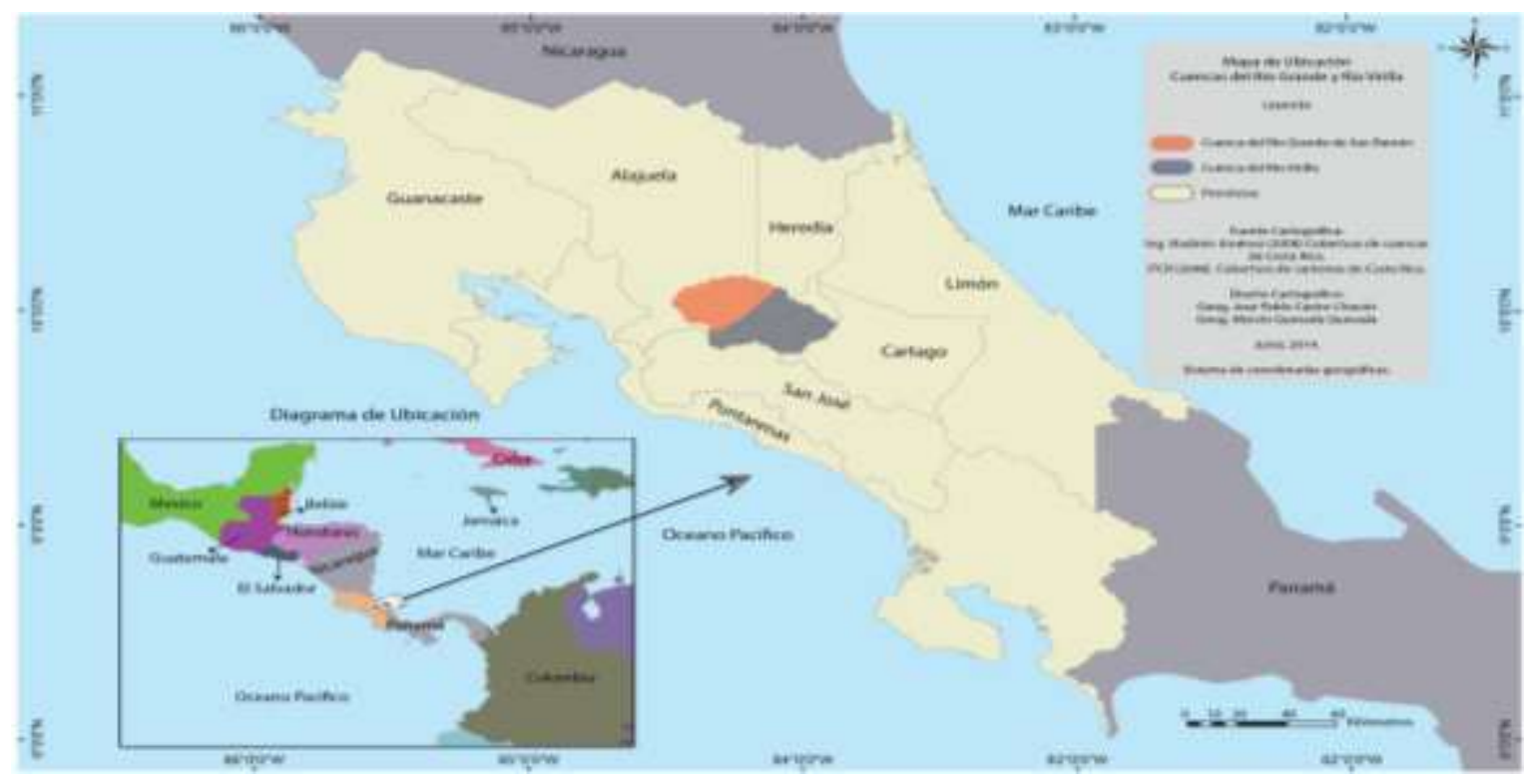

Fuente: Elaboración propia con base en el Atlas Digital de Costa Rica, 2008.

\section{CONDICIONES CLIMÁTICAS Y SU RELACIÓN CON EL ENOS}

Desde el punto de vista climatológico, en la vertiente Pacífica de Costa Rica, lugar donde se ubica la cuenca del río Tárcoles, la influencia de los vientos Alisios más intensos ocurren durante el período seco (diciembre a marzo). Sin embargo, de acuerdo con algunas investigaciones realizadas en Costa Rica, dicha influencia no solo ocurre durante ese período seco. Por ejemplo, FERNÁNDEZ y RAMírEZ (1991) aseguran que durante los años de la fase cálida del ENOS (El Niño), se han observado vientos Alisios del Noreste que ingresan por el sector Caribe, los cuales son más fuertes en algunos meses de la estación lluviosa (mayo a octubre) e incluso durante algunos años como en 1972 esos vientos persistieron durante gran parte del año.

Por lo tanto, en esos años de la fase cálida, el régimen de precipitación se altera con respecto a sus valores normales, tanto en su cantidad como en su distribución (Quesada y Waylen, 2013). Cuando es un año El Niño, la estación lluviosa es irregular y la estación seca siguiente es más cálida y seca que en los años en que ocurre la fase fría (La Niña), en la cual las condiciones parecieran ser totalmente opuestas, mientras que durante los años normales las precipitaciones son más estables, no se presentan diferencias extremas en los niveles de 
caudal en la vertiente Pacífica (GEORGE ET AL., 1998).

La disminución de la precipitación en la vertiente Pacífica durante los años de la fase cálida del ENOS pareciera estar asociada con la disminución en la velocidad de los vientos provenientes del Oeste (FERNÁNDEZ Y RAMÍREZ, 1991) y por la influencia de patrones de circulación global (WAYLEN Y OTROS, 1996). Esta irregularidad en la precipitación afecta directamente las descargas de los ríos (GEORGE ET AL., 1998; W AYLEN AND LAPORTE, 1999), causando pérdidas considerables en aquellas regiones del país que requieren agua de los ríos para realizar varias actividades indispensables para el ser humano, como son la irrigación, la hidroelectricidad, el consumo doméstico, industrial, comercial, la pesca, abrevaderos, e incluso el campo del turismo se ve afectado.

De ahí que analizar los cambios en la precipitación en la Depresión Tectónica Central en forma anual y estacional tenga una gran importancia socioeconómica, dado que todas las actividades mencionadas en el párrafo anterior se desarrollan en esta región.

\section{LA PRECIPITACIÓN EN LA DREPRSIÓN TECTÓNICA CENTRAL Y SU RELACIÓN CON EL ENOS}

La variabilidad climática que se subsista cuando se empieza a desarrollar el evento el ENOS consiste en lo siguiente: Se inicia a través de los cambios en las temperaturas superficiales del océano Atlántico (TSOA) y del océano Pacífico (TSOP), las cuales varían unos grados centígrados por arriba o debajo de lo normal (POVEDA G., P.R. WAYLEN \& R.S. PULWARTY, 2006) . Dichos cambios podrían explicar las tendencias que han tenido los caudales en la Depresión Tectónica Central, dado que cuando esa situación ocurre, hay variaciones en la circulación y dirección de los vientos, así como en la presencia de disturbios atmosféricos (WAYLEN AND QUESADA, 2002). Es sabido que a mediados de los años setenta ocurrió un cambio climático a nivel mundial, lo cual se visualiza en la siguiente gráfica (IPCC, 2007; HOUGHTON et al., 2012; COAPS, 2012) (Figura 2) 
Figura 2. Serie cronológica del IOS y las TSOA

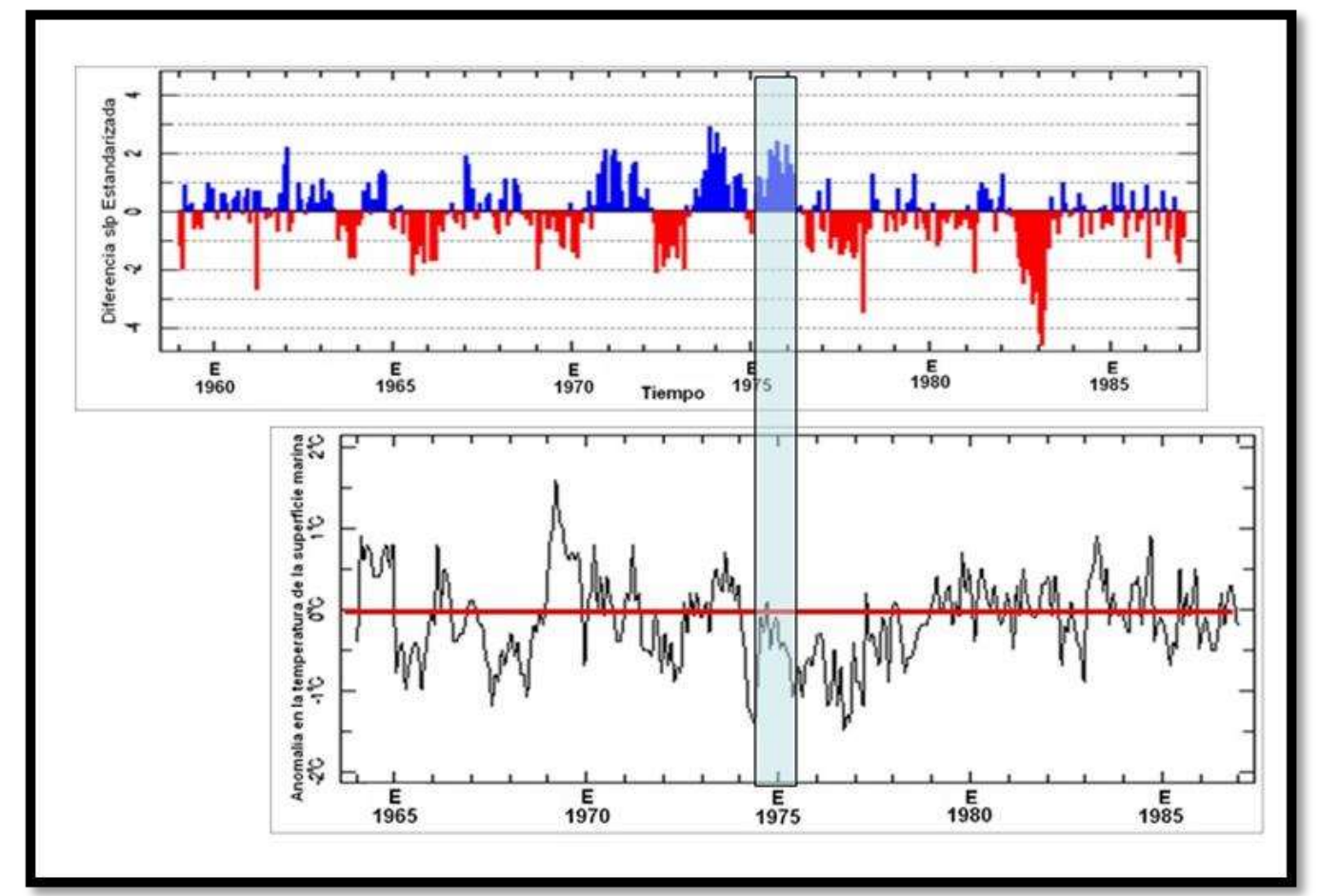

Fuente: http/www.nnic.noaa.gov/data/indices

En la serie cronológica disponible sobre el Índice de Oscilación del Sur (IOS) (Figura 2) se visualizan tanto, eventos El Niño (Fase Cálida), como eventos La Niña (Fase Fría). Como se puede observar en la gráfica en el océano Atlántico ocurrió un cambio cerca de 1975, con anomalías positivas tempranas en las (TSOA) y anomalías negativas tardías después de 1975. A la hora de interrelacionar el IOS con las temperaturas superficiales de ambos océanos se ha encontrado que existe una fuerte influencia sobre la precipitación de la región Pacífica de Costa Rica (Poveda AND MeZA, 1997).

Los análisis de las desviaciones estándar de la precipitación estacional en el contexto de un simple balance de agua podría dilucidar la compleja interacción entre la precipitación y la variabilidad climática.

\section{MÉTODO DE ANÁLISIS}

Con la idea de lograr un estudio exhaustivo del comportamiento de la precipitación en la Depresión Tectónica Central (Subcuencas ríos Virilla y Grande de San Ramón), se 
efectuó un análisis desde el punto de vista estacional, tomando como referencia cinco períodos a través del año, los cuales corresponden con el comportamiento de los principales patrones de circulación general que afectan el área en estudio. Estos se detallan a continuación:

a) EFMA. Este período se manifiesta a partir del mes de Enero hasta Abril y coincide con la incursión de los vientos fríos llamados Los Nortes, los cuales aparentemente son más frecuentes en Costa Rica durante la fase cálida del ENOS.

b) MJ. Este período incluye Mayo y Junio, que precisamente coinciden con la primera máxima de precipitación en la vertiente Pacífica de Costa Rica.

c) JA. Incluye los meses de Julio y Agosto. Aunque se debe considerar a partir de la última semana de junio (veranillo de San Juan), una semana de julio y otra de agosto en años normales. En estos meses se produce una merma en las lluvias en el sector pacífico de casi toda la América Central (MAGAÑA Y OTROS, 1999). La duración de estos períodos depende de la fase en la que se encuentra el evento el ENOS.

d) SO. Este período se manifiesta durante Septiembre y Octubre en el área de estudio y corresponde con uno de los períodos de mayor influencia de la Zona de Convergencia Intertropical, es precisamente cuando se registran las máximas precipitaciones en la región en estudio.

e) ND. Este período se manifiesta entre Noviembre y Diciembre, siendo el primero de estos meses, el de la transición entre la época lluviosa y la seca. Mientras que el segundo mes se caracteriza por las temperaturas frías y a veces acompañados de lloviznas.

Para efectos de lograr un mayor detalle geográfico en el análisis de la precipitación en el área en estudio se dividió la Depresión Tectónica Central en dos subcuencas la del río Virilla y la del río Grande de San Ramón.

La desviación estándar fue calculada para cada Estación Meteorológica utilizadas en la investigación y luego se correlacionó con las fases cálida y fría que muestran los océanos Atlántico y Pacífico. Esta correlación muestra las condiciones oceánicas que ocurren en cada uno de los periodos que se detallaron anteriormente. 
En síntesis, este análisis indica la magnitud de la interacción que se da entre las temperaturas superficiales del mar (TSM) en los océanos Atlántico y Pacífico y su influencia en la distribución estacional de la precipitación en ambas subcuencas de estudio. Para hacer la interpolación de las variables se utilizó el Método Inverso a la Distancia.

Las TSM desde 1950 hasta el 2010 se analizaron para el Océano Atlántico Norte y el Océano Pacífico Ecuatorial (Figura 2). Mientras que directamente en la región en estudio se consideraron todas aquellas Estaciones Meteorológicas con registro de precipitación mensuales superiores a veinte años completos. A todas las Estaciones Meteorológicas se les calculó la media y la desviación estándar. Cada uno de los registros disponibles de cada serie es subdividida en cuatro categorías dependiendo de la anomalía de las TSM (positivas o negativas) en los dos océanos según el periodo de análisis (EFMA, MJ, JA, SO, ND).

Con la idea de tener una comparación geográfica de los promedios de cada una de las series temporales se comparan con cada una de las condiciones oceánicas citadas. Estas son expresadas como una desviación normal estándar y se relacionan con los promedios de cada Estación Meteorológica para comparar los resultados de las precipitaciones bajo diferentes combinaciones de condiciones oceánicas. De esta forma, se obtiene si una condición es más húmeda o más seca que la otra.

\section{FLUCTUACIONES EN LAS TEMPERATURAS MARINAS DURANTE LAS FASES DEL ENOS}

Los registros en la precipitación entre 1959-2010 muestran una baja precipitación en el período A (EFMA) para la subcuenca del río Grande de San Ramón y una ligera desviación positiva para la subcuenca del río Virilla. Los registros de precipitación estacional tienen muy poco impacto en las descargas de los ríos, dado que en este momento en ambas subcuencas se desarrolla un periodo seco. Sin embargo, si se analiza detenidamente de acuerdo con la fase del ENOS se tiene que durante esa época cuando existen un Pacífico Frío con un Atlántico Frío, existe la tendencia a tener condiciones más húmedas (ciertos días con lloviznas) en ambas subcuencas, especialmente en la del río Grande de San Ramón.

Cuando el Pacífico esta frío y el Atlántico cálido, en el área en estudio se dan 
condiciones intermedias entre lluvioso y seco, siendo un poco más lluviosa la parte alta de la cuenca. Sin embargo, al analizar el registro histórico antes de 1975 cuando se tenía un Pacífico cálido con Atlántico cálido, se manifestaron condiciones más lluviosas en ambas subcuencas. En tanto, posterior a 1975 cuando el Pacífico estaba cálido y el Atlántico frío se manifestaron condiciones aún más secas, sobre todo en la subcuenca del río Grande de San Ramón.

La variabilidad climática puede ser explicada al combinarse las anomalías en las temperaturas superficiales del mar en ambos océanos con el efecto que puede producir el evento de ENOS. Es conocido que a partir del año de 1975 se dio un cambio trascendental al aumentar las temperaturas ambientales, las cuales es catalogada por algunos científicos como de tres veces superior a la ocurrida durante los últimos cien años (Houghton et al., 2011) (Figura 3). El IPCC en su informe del 2007 avala que los fenómenos meteorológicos han cambiado de frecuencia y/o intensidad en los últimos cincuenta años, por ejemplo es probable que lo días fríos, las noches frías y las escarchas sean ahora menos frecuentes en la mayoría de las regiones terrestres, mientras que los días y noches cálidos serían ahora más frecuentes. Las observaciones evidencian un aumento de la actividad ciclónica tropical intensa en el Atlántico Norte desde 1975, y parecen indicar un aumento de esa actividad en algunas otras regiones en que la calidad de los datos es más dudosa (IPCC, 2007).

Esta situación también se muestra en el Índice de Oscilación del Sur. Es así como anterior a 1975 se nota cierta predominancia de las fase fría del ENOS. En tanto, después de 1975, la fase cálida tiende a dominar. Es así como el océano Atlántico muestra un cambio cerca del año 1976, siendo predominantemente negativa en sus temperaturas superficiales y una débil magnitud positiva posterior a 1976, lo que indica que predomina un océano Atlántico frío. De esta forma, cuando se tiene una combinación de un océano Atlántico frío con un océano Pacífico cálido se produce una gran variabilidad climática lo cual influye en la cantidad de precipitación que se presenta en ambas subcuencas en estudio. 
Figura 3. Cambio de la Temperatura Mundial y Continental

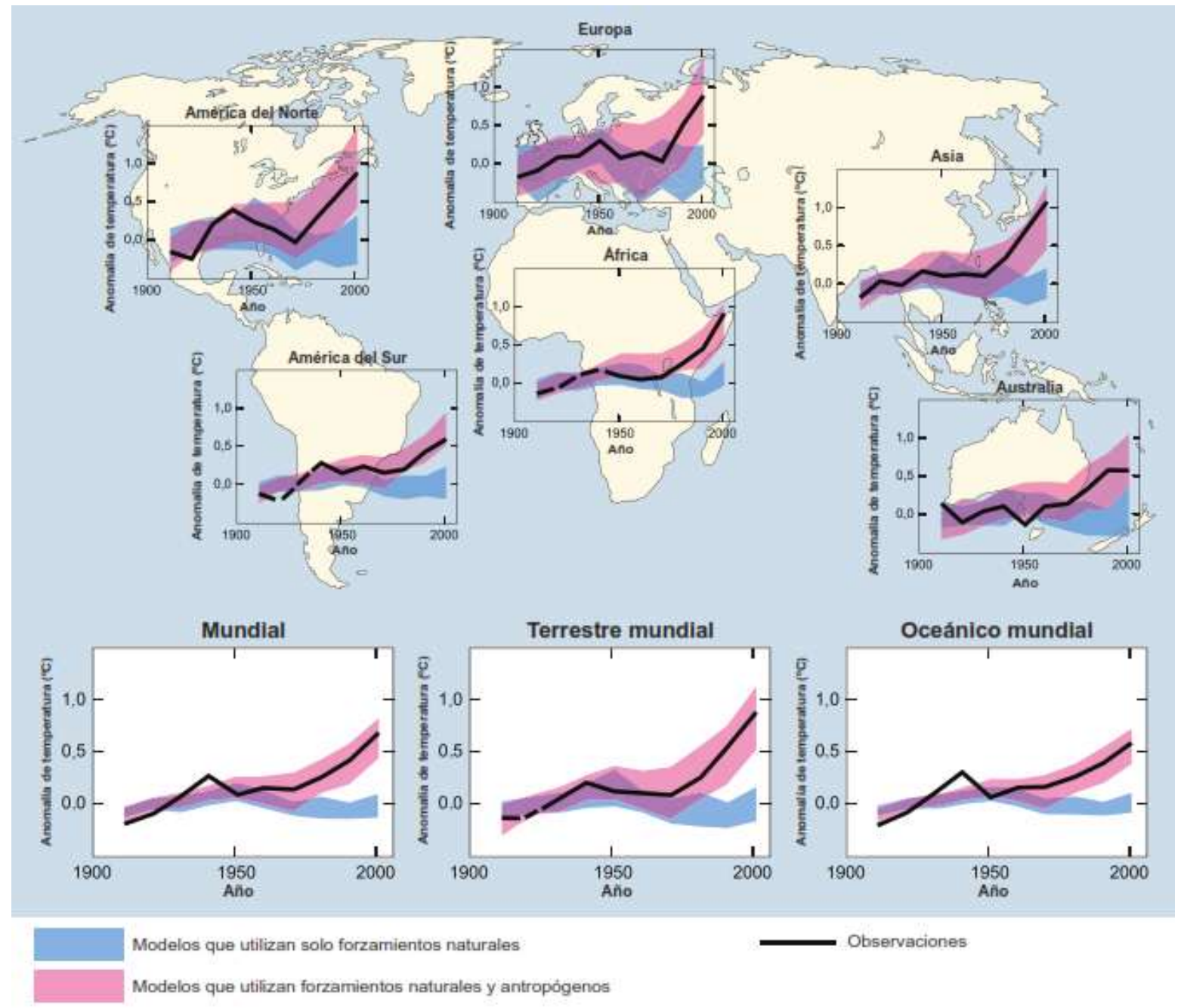

Fuente: IPCC, 2007 
Figura 4. Precipitación en diferentes condiciones oceánicas en la Depresión Tectónica Central en el periodo de EFMA
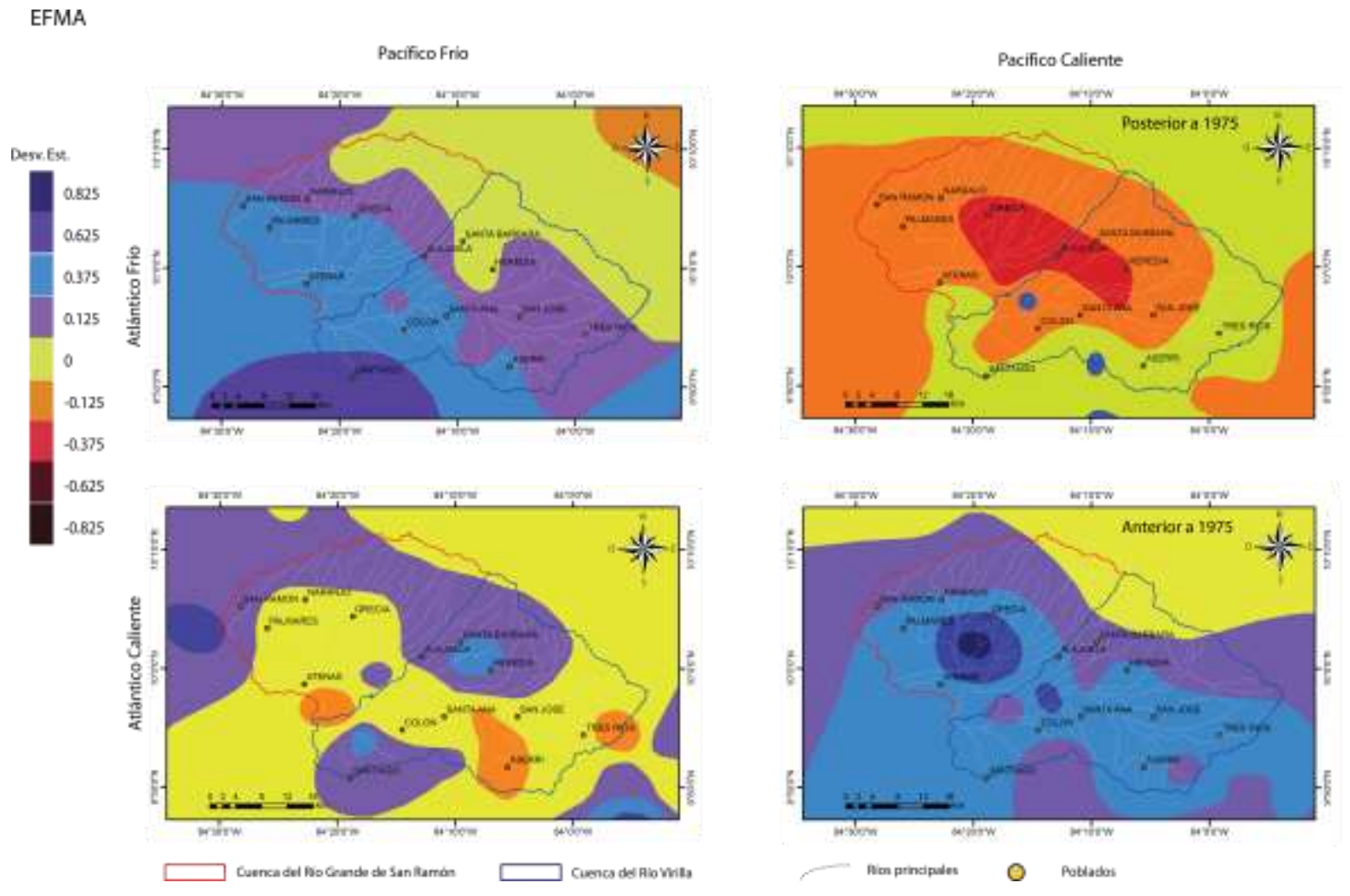

Fuente: Elaboración propia con base en los registros de precipitación suministrados por el Instituto Meteorológico Nacional de Costa Rica, 2009.

El grafico anterior muestra la Depresión Tectónica Central dividida en dos sectores: Al lado derecho la subcuenca del río Virilla y al lado izquierdo la subcuenca del río Grande de San Ramón.

Para el periodo B (MJ) también ocurren desviaciones negativas en la precipitación debido a la combinación de un Pacífico cálido con un Atlántico frío. La disminución en la precipitación en la subcuenca del río Grande de San Ramón, se produce en el sector Suroeste de la subcuenca y podría deberse a las características topográficas de la cuenca. Bajo dichas condiciones se da un efecto de sotavento, lo que conduce, más tarde, a la producción de menos lluvias. 
Figura 5. Precipitación en la condición Pacífico cálido y Atlántico frío en la Depresión Tectónica Central para el periodo de Mayo - Junio.

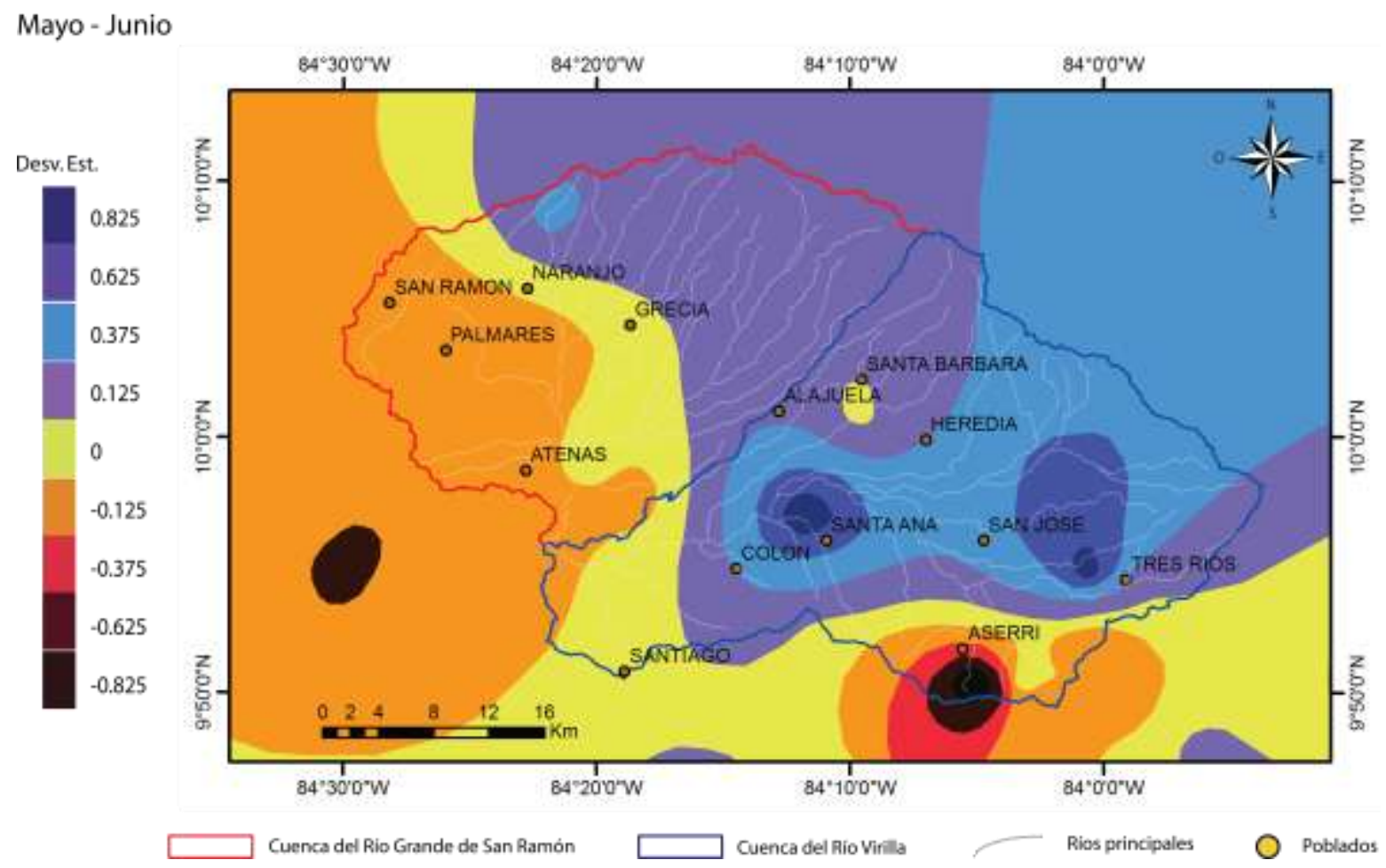

Fuente: Elaboración propia con base en los registros de precipitación suministrados por el Instituto Meteorológico Nacional de Costa Rica, 2009.

El periodo C (JA) (Figura 6) que se distingue normalmente por la presencia del veranillo de San Juan y las canículas. Este periodo ha mostrado ser particularmente muy sensible a la señal de ENOS durante su fase cálida (W AYLEN AND QUESADA, 2002). Cuando se manifiesta un Pacífico cálido con un Atlántico frío se da una señal de merma en la precipitación que influye, aún más, sobre la subcuenca del río Grande de San Ramón. Esto por cuanto esta subcuenca se encuentra a sotavento de la Sección de la cordillera Central, aspecto que induce a que se produzcan condiciones más secas.

La disminución en la precipitación en esos meses se da por el alejamiento de la Zona de Convergencia Intertropical hacia el Norte de las latitudes a las que se encuentra Costa Rica. Esto permite que incursione con más intensidad los vientos Alisios del Noreste, ocasionando un efecto de subsidencia sobre ambas subcuencas y sobre todo en la subcuenca del río Grande de San Ramón, en aquellos sitios donde existen valles y planicies como es el 
caso de Palmares, Atenas, Grecia, La Garita, Turrucares, La Guácima, Belén, Alajuela, entre otros.

En este periodo se da una amplia respuesta a la condición de Atlántico frío con un Pacífico cálido, ya que se incrementa el valor de las desviaciones estándar, mostrando una fuerte señal de merma en la precipitación en ambas subcuencas, especialmente en la subcuenca del río Grande de San Ramón.

Figura 6. Precipitación en la condición Pacífico cálido y Atlántico frío en la Depresión Tectónica Central para el periodo de Julio - Agosto

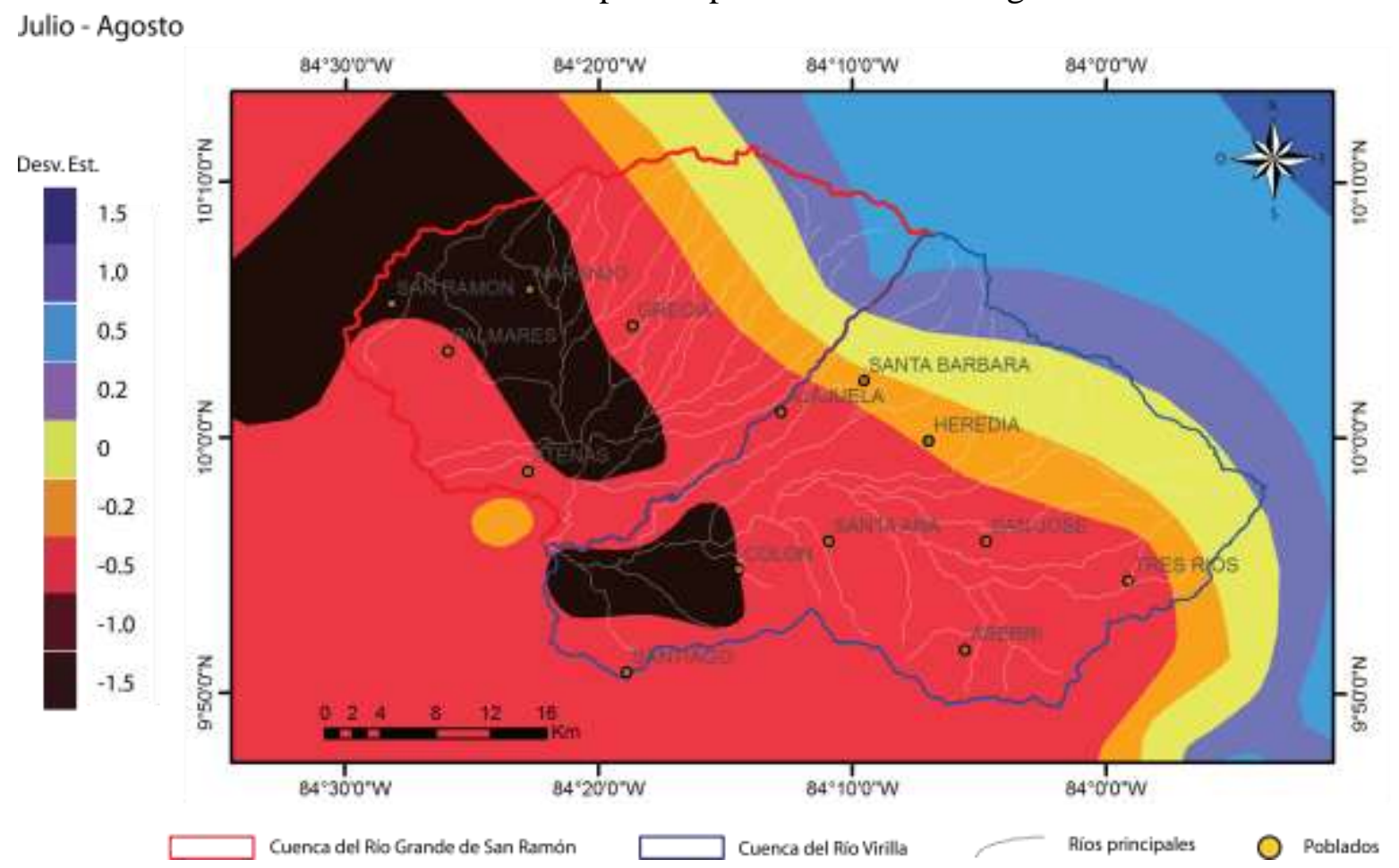

Fuente: Elaboración propia con base en los registros de precipitación suministrados por el Instituto Meteorológico Nacional de Costa Rica, 2009.

Durante el periodo D (SO) (Figura 7) persiste una condición de una ligera merma en las lluvias en la subcuenca del río Grande de San Ramón, a pesar de ser meses muy lluviosos. La subcuenca del río Virilla no muestra condiciones tan secas, dada la incursión de masas de aire cargadas de humedad provenientes del Caribe que logran cruzar el sistema montañoso a través del paso intermontano de La Palma. Aunque, la subcuenca del río Grande de San Ramón muestra una mayor respuesta ante dicha condición del evento El Niño, 
que la del Virilla, las descargas no reflejan que los suelos estén saturados de agua. De ahí que ambas subcuencas tengan una respuesta muy similar (evidenciado por la no linealidad de la respuesta de las subcuencas).

Figura 7. Precipitación en la condición Pacífico cálido y Atlántico frío en la Depresión \begin{tabular}{l} 
Septiembre \\
\hline 0.825 \\
0.625 \\
0.375 \\
0.125 \\
0 \\
\hline-0.125 \\
-0.375 \\
-0.625 \\
\hline-0.825
\end{tabular} Tectónica Central para el periodo Septiembre - Octubre.

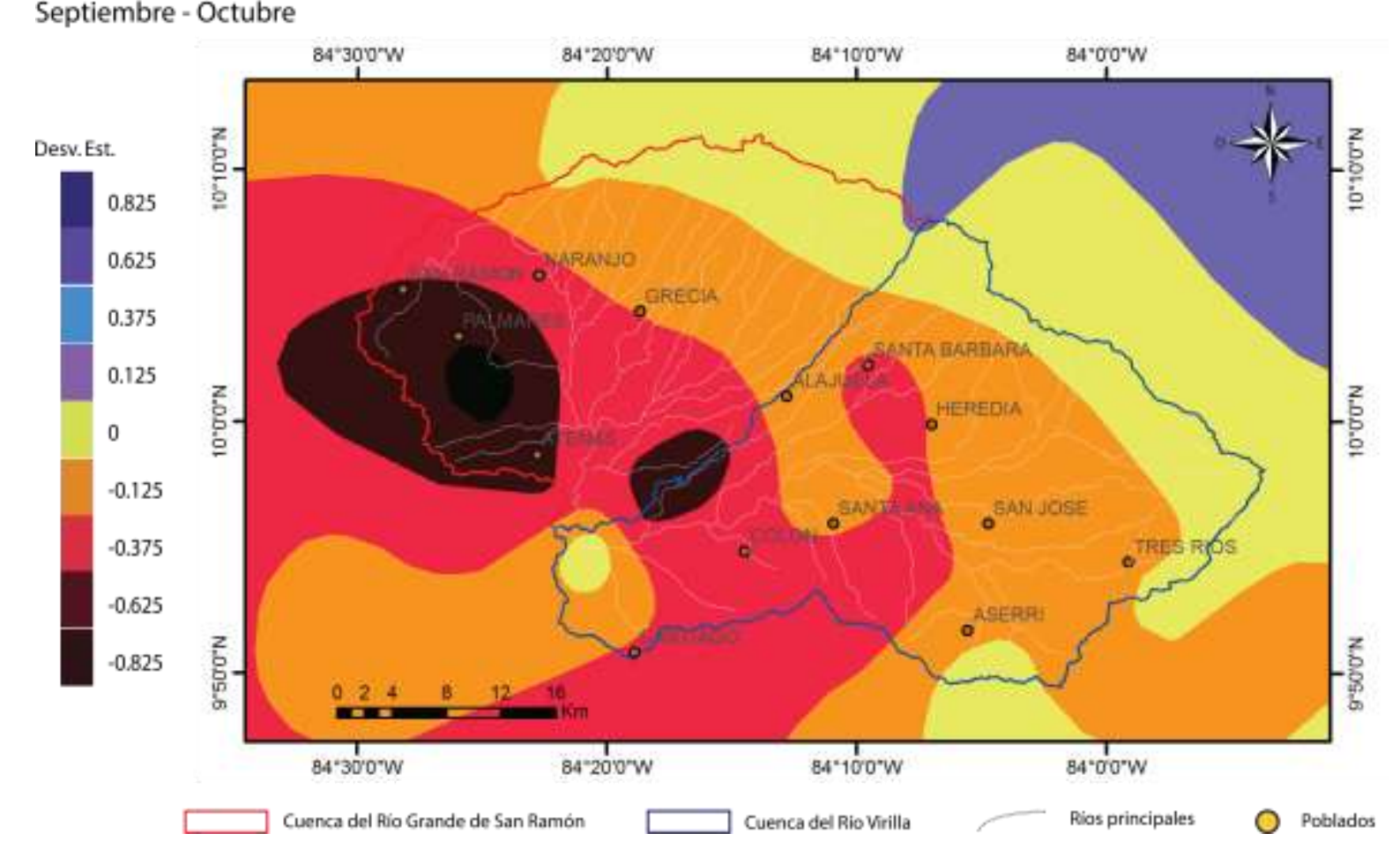

Fuente: Elaboración propia con base en los registros de precipitación suministrados por el Instituto Meteorológico Nacional de Costa Rica, 2009.

En el periodo $\mathbf{E}$ (ND) (Figura 8) la señal que se manifestó en los meses previos cambió en ambas subcuencas, donde las condiciones pasan de ser secas, a convertirse en relativamente más húmedas. Se da una respuesta positiva ante el ENOS pero con mayor influencia del océano Atlántico Norte. En la subcuenca del río Grande de San Ramón no se muestra ningún caudal significativo, sobre todo porque la estación lluviosa en esos meses está terminando. En tanto, el río Virilla tiene una desviación estándar superior, o sea una presencia mayor de precipitación, la cual se explica, como ya se señaló, por la presencia del paso intermontano o depresión de la Palma, lugar por donde lograr pasar masas de aire cargadas de humedad aspecto que se visualiza con mayor claridad en los cerros de 
La Carpintera, cerca del cerro de Ochomogo en Cartago.

Este paso facilita la incursión de los vientos Los Nortes (W AYLEN ET AL., 1998) que proceden de Norte América. Algunas estaciones meteorológicas ubicadas en la parte media baja de la subcuenca del río Grande de San Ramón (Fabio Baudrit, Sabana Larga) muestran condiciones de altas precipitaciones durante los meses de Noviembre y Diciembre. Esto es producto de masas de aire provenientes del Caribe y que logran incursionar por dichos pasos intermontanos y que por la ubicación de estas estaciones (zona de confluencia de vientos) se logra producir una mayor presencia de lluvias.

Figura 8. Precipitación en la condición Pacífico cálido y Atlántico frío en la Depresión Tectónica Central para el periodo Noviembre - Diciembre.

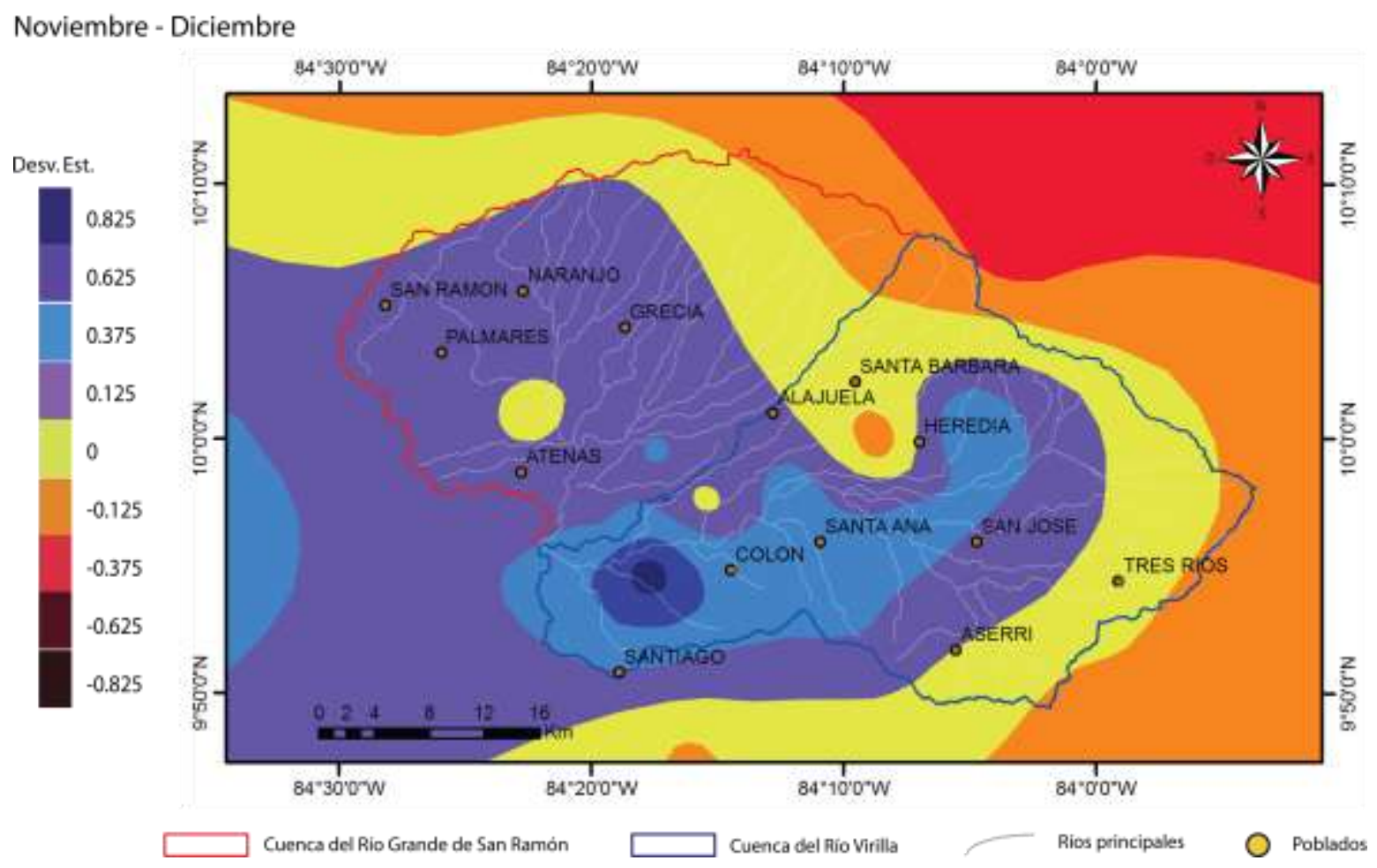

Fuente: Elaboración propia con base en los registros de precipitación suministrados por el Instituto Meteorológico Nacional de Costa Rica, 2009. 


\section{CONCLUSIÓN}

En síntesis, los análisis efectuados indican que ambas subcuencas muestran una declinación en la cantidad de precipitaciones anuales a partir del 1975. Esta declinación es aún mayor en la subcuenca del río Grande de San Ramón en comparación con la subcuenca del río Virilla. Este declive en la precipitación a partir del año 1975 se encuentra relacionado con la fase cálida del ENOS, al estar el océano Pacífico con condiciones de aguas cálidas, se inhibe la incursión de los vientos provenientes del sector Pacífico y que normalmente influyen entre Mayo y Octubre sobre la vertiente Pacífica de Costa Rica. Unido a ello, la presencia de un océano Atlántico Frío contribuye con una menor presencia de lluvias sobre la región en estudio.

A pesar de que ambas subcuencas se ubican una a la par de la otra, la respuesta ante el evento del ENOS es diferente, dada la diferencia de temperaturas entre un océano y otro, lo que conlleva a una menor incursión de vientos que contribuyen en la producción de masas de aire cargadas de humedad que puedan llegar hasta la Depresión Tectónica Central, provenientes del sector Pacífico.

Similarmente, las irregularidades geomorfológicas de ambas subcuencas contribuyen aún más con las diferencias en los niveles de precipitación en las subcuencas, siendo esta reducción mayor en la subcuenca del río Grande de San Ramón. En tanto, en la subcuenca del río Virilla presenta mayores niveles de precipitación, especialmente porque presenta en su sector Noreste una depresión intermontana que permite la incursión de masas de aire cargadas de humedad provenientes de la vertiente Caribe.

Además, se encontró que cuando ambos océanos Pacífico y Atlántico presentan temperaturas frías, las condiciones son húmedas en las subcuencas, presentándose mayor precipitación en toda la zona. En el caso que la condición del Pacífico sea fría y el Atlántico caliente, provoca que en la zona de estudio haya una diferencia notoria de precipitación entre una subcuenca y la otra; en este aspecto la subcuenca del Río Grande de San Ramón presenta mayor precipitación en el sector Oeste-alto. Sin embargo en sus zonas intermedias y bajas hay una disminución de humedad y de lluvias. Estas alteraciones, como se ha mencionado anteriormente es resultado del cambio vertiginoso acontecido a partir de 1975, ya que antes de ese año las subcuencas presentaban mayor concentración de humedad. Después de 1975, 
la temperatura tanto ambiental como del océano Pacífico, aumentaron ocasionando una disminución en la humedad.

Durante el periodo de Mayo-Junio, la humedad es mayor debido a la movilización de los vientos provenientes del Caribe entre el paso intermontano de la Palma de la Depresión Tectónica Central, sin embargo es notoria una disminución de las precipitaciones en el sector oeste de la subcuenca del Río Grande de San Ramón.

A diferencia del periodo anterior donde la humedad de las subcuencas es mayor, durante Julio-Agosto en la subcuenca del Virilla, dentro de su sector inferior-Oeste hay un descenso en los niveles de precipitación. En el caso de la subcuenca de San Ramón se da una abrupta disminución en su sector Oeste, de ese modo siendo notorio el evento del ENOS.

Por último, el periodo Noviembre-Diciembre presenta una estabilización en las precipitaciones y en la humedad, donde se nota que el periodo del evento del ENOS disminuye su influencia.

Aunque no se analizó en esta investigación, se puede constatar que el uso de la tierra existente en ambas subcuencas ha mostrado cambios durante los años, siendo la subcuenca del río Virilla la que ha presentado un cambio más radical al ser una región muy poblada, caracterizada por las infraestructuras, pavimentos y demás áreas que imposibilitan la infiltración de las aguas de lluvia.

La presión por ocupar el espacio con fines residenciales es muy fuerte, lo que ha ocasionado que en periodos de lluvias, estas escurran rápidamente hacia los cursos de los distintos ríos de esta subcuenca. La presencia de tanta masa urbana podría estar contribuyendo a que durante días cálidos, la evaporación sea alta y coadyuve a la formación de nubes cumulonimbos que luego precipitan en forma muy intensa, en sectores circunvecinos.

En tanto, en la subcuenca del río Grande de San Ramón, los sectores con urbanizaciones han incrementado pero en menor grado, de ahí que no se tengan cambios drásticos en los niveles de precipitación. 


\section{AGRADECIMIENTO}

Se agradece a la Vicerrectoría de Investigación por el apoyo brindado en el financiamiento del Proyecto de Investigación de la Universidad de Costa Rica. N 540-A7-027. Así como al profesor Peter R. Waylen por sus sugerencias y comentarios.

Asimismo, se agradece a los estudiantes Keneth Murillo y Pablo Castro, ambos de la Universidad de Costa Rica, por la colaboración en la elaboración de mapas, figuras y tablas.

\section{REFERENCIAS BIBLIOGRÁFICAS}

CAVAZOS, T., GOCHIS, D., MARENGO, J., \& BOULANGER, J.-P. 2008. VAMOS and Anthropogenic Climate Change (ACC) In the Americas.World Climate Research Programme. CLIVAR Noviembre.Project. Obtenido de http://www.clivar.org/organization/vamos/resources

COAPS, 2012. ENSO Index According to JMA SSTA (1868-present). Florida, Center for Ocean-Atmospheric Prediction Studies. The Florida State University. Disponible en: http://coaps.fsu.edu/jma.shtml.

DURAN A., J. GEMENO, J.A. AMADOR \& R. NIETO, 2010. Moisture Sources for Central America: Identification of Moisture Sources using a Lagrangian Analysis Technique. Journal of Geophysical Research. 115: 5-103.

Enfield, D.B. AND E.J. Alfaro. 1999. The Dependence of Caribbean Rainfall on the Interaction of the Tropical Atlantic and Pacific Oceans. Journal of Climate, 12, 20932103.

ENRIQUEZ, O., GUZMAN, A., \& NARVÁEZ, G. 2014. Análisis del comportamiento de la precipitación en el municipio. Obtenido de Cuadernos de Geografía. Enero-Junio.

FERNÁNDEZ W, RAMíreZ P. 1991. EL Niño. La Oscilación del Sur, sus efectos sobre Costa Rica: Una revisión. Tecnología en Marcha. Vol. 11: 3-11.

GEORGE, R ET AL. 1998. Interannal variability of annual streamflow and the Southern Oscillation in Costa Rica. Journal of Hydrological Sciences. 43 (3). http/www.nnic.noaa.gov/data/índices.

HASTENRATH SL. 1990. Variations in low latitude circulation and extreme climatic events in the tropical Americas. Journal of the Atmospheric Sciences 33: 202-215. 
HOUGHTON et al., 2012. Carbon emissions from land use and land-cover change. Biogeosciences, 9, 5125-5142, 2012

IPCC, 2007: Cambio climático 2007: Informe de síntesis. Contribución de los Grupos de trabajo I, II y III al Cuarto Informe de evaluación del Grupo Intergubernamental de Expertos sobre el Cambio Climático [Equipo de redacción principal: Pachauri, R.K. y Reisinger, A. (directores de la publicación)]. IPCC, Ginebra, Suiza, 104 págs.

Magaña, V., J. A. Amador And S. Medina. 1999. The midsummer drought over Mexico and Central America, Journal of Climate, 12(6), 1577-1588.

MURPHY J., K. GEORGAKAKOS \& E. SHAMIR, 2013. Climatological Analysis of December Rainfall in the Panama Canal Watershed. International Journal of Climatology., doi: 10.1002/joc.3694.

POVEDA G., O.J. MESA, L.F. SALAZAR, P.A. ARIAS, H.A. MORENO, S.C. VIEIRA, P.A.GUDELO, V.G. TORO \& J.F. ALVAREZ, 2005. The Diurnal Cycle of Precipitation in the Tropical Andes of Colombia. Monthly Weather Review. 133 (1), 228-240.

POVEDA G., P.R. WAYLEN \& R.S. PULWARTY, 2006. Annual and Inter-annual Variability of the Present Climate in Northern South America and Southern Mesoamerica. Palaeogeography, Palaeoclimatology, Palaeoecology. 234: 3-27.

QUESADA, M \& WAYLEN, P. 2014. Análisis climático de la precipitación anual e interanual en la cuenca media del río Grande de San Ramón, Costa Rica (Vol. 45). (I. Geográficas, Ed.) Santiago, Chile: Investigaciones Geográficas.

YOULTON, C., SHIRATSUBAKI, T., TARSE SANCHES DE OLIVEIRA, P., \& WENDLAND, E. (2012). Influencia del fenómeno El Niño-La Niña y cambio (Vol. LXXIII). Sao Pablo: Estudios Geográficos. doi:10.3989/estgeogr.201212

WAYLEN, P., M. QUESADA. 2002. The effect of Atlantic and Pacific sea surface temperatures on the mid- summer drought of Costa Rica, Environmental change and Water Sustainability, Instituto Panamericano de Ecología, Zaragoza.

WAYLEN, P. ET AL. 1998. Rainfall distribution and regime in Costa Rica and its response to the El Nino- Southern Oscillation, Conference of Latin Americanist Geographers Yearbook, 24 (1998) p 75-84, 75-84. 
WAYLEN, P AND S, LAPORTE. 1999. "Flooding and the El Niño-Southern Oscillation phenomenon along the Pacific coast of Costa Rica." Hydrological Processes, 13, 26232638.

WAYLEN P. R. \& M.E. QUESADA, 2002. The Effect of Atlantic and Pacific Sea Surface Temperature on the Mid- Summer Drought of Costa Rica. Journal of Environmental Change and Water Sustainability. Instituto Pirenaico de Ecología, Zaragoza. España. 197-209. 